\title{
Liderazgo y procesos de mejora de la educación superior
}

El liderazgo en las universidades

Fabrina Monserrate Cedeño Mendoza Mg. Sc..

Instituto Tecnológico Superior Portoviejo, Ecuador

Receptado: 17/07/2014 Aceptado: 04/09/2014

Contacto: $\underline{\text { fcedeno@itsup.edu.ec }}$

\section{Resumen}

El trabajo aborda la incompatibilidad existente en el plano del liderazgo y la mejora de los profesores en los diferentes procesos y tareas de la educación superior, que se visualiza desde diferentes ángulos. Por una parte, los modos de crear el cambio educativo y las reformas, por otro, los disímiles enfoques sobre las organizaciones educativas convenientes con los modos de pensar, así como las distintas ideas de la profesionalidad docente y el ejercicio de la enseñanza. Este trabajo es parte de una investigación realizada en el Instituto Tecnológico Superior Portoviejo, Ecuador, que tuvo como objetivo proponer vías para la configuración de centros educativos superiores como comunidades profesionales de instrucción que puedan posibilitar el aprendizaje a través del trabajo conjunto. Para corroborar los resultados de este, se aplicaron encuestas a profesores que laboran en este nivel de enseñanza. Mediante las deducciones obtenidas, se concluyó que la capacidad para mejorar una institución de educación superior depende, de manera relevante, de equipos directivos con liderazgo que contribuyan a dinamizar, apoyar, animar los procesos sustantivos con un buen nivel de eficacia que contribuya a la capacidad interna de mejora; lo que se precisa una eficaz formación de estos.

Palabras claves: aprendizaje; cambio educativo; enseñanza; liderazgo; mejora

\section{Leadership and process improvement of higher education}

\begin{abstract}
The paper states the incompatibility therein in the field of leadership and improvement of professors in the different processes and tasks of higher education, which is

viewed from different points. On the one hand, the ways of creating educational changes and reform, on the other, dissimilar approaches to educational organizations, convenient ways of thinking and different ideas of teacher's professionalism and the academic exercise as well. This work is part of research carried out at the "Instituto Tecnológico Superior Portoviejo, Ecuador, which aimed to propose ways for setting higher schools as communities that might enable to work with the learning through working together. To corroborate the results, surveys were applied to teachers working at this level. By the deductions obtained, it was concluded that the ability to enhance an institution of higher
\end{abstract}


Rev. SINAPSIS, Vol. 5, N² 2, Diciembre 2014

ISSN $1390-7832$

education depends, in a relevant way of management with leadership that helps stimulate, support, encourage and substantive processes with a good level of efficiency that supports capacity and internal improvement; for which it is required an effective training .

Keywords: learning, educational change, education, leadership, improvemt

\section{Introducción}

La construcción de este artículo se enmarca dentro de un proyecto de investigación de análisis social-educativo con el apoyo facultativo del personal docente y administrativo de las distintas universidades e institutos de la Provincia de Manabí denominado "LIDERAZGO Y MEJORA DE LA EDUCACIÓN SUPERIOR", "Liderazgo es influencia. Esa es una definición exacta y acertada de lo que es liderazgo. Sin influencia no existe liderazgo" Arana (2013p.3) .Esta es realidad a la que tienen que enfrentarse los centros de educación superior por la heterogeneidad de las demandas derivadas de las reformas educativas, del entorno local y de los agentes implicados y su influencia en los subordinados.

La gran necesidad de permanecer en un mundo cada día más exigente y globalizado, ha obligado a instituciones de educación superior a realizar cambios en su estructura, orientados a mejorar la calidad de sus procesos. En este sentido el liderazgo se ha convertido en una herramienta útil para la gerencia universitaria, en la búsqueda por alcanzar el logro de sus objetivos.

Cualquier propuesta medianamente compleja sobre el liderazgo y mejora de la educación superior debiera situarse entre concebirlo como un gerente eficiente, identificado con el enfoque formal ocupada por una persona; y como labor moral amplia, expresión cualitativa de una organización dinámica y comunitaria, y como tal, una función difusa entre todo el profesorado. El problema de un discurso crítico por tanto, con claros referentes transformadores de la realidad se sitúa, pues, entre la visión profesionalizada, y las propuestas de las organizaciones educativas en que las funciones del liderazgo sean compartidas y dispersas. El problema al que nos enfrentamos es, cómo hacer compatible el liderazgo y mejora de los profesores, en diferentes espacios y tareas.

El tema del liderazgo y mejora de la educación superior se presenta, entonces, con una mezcla de planos y ángulos, que impiden una reflexión seria, sobre todo cuando se sustancializa, en lugar de registrarlo en el marco de los centros como organizaciones y en el contexto de perfeccionamiento. Un modo para situar las distintas visiones del liderazgo y mejora en la 
educación superior, al tiempo que para comprenderlo como algo multidimensional, es el cruce entre: por una parte, los modos de crear el cambio educativo y las reformas, por otro, los disímiles enfoques sobre las organizaciones educativas, convenientes con los modos de pensar de la enseñanza. Unido a ellos, las distintas ideas de la profesionalidad docente y el ejercicio de la enseñanza.

Tomando como problema la incompatibilidad existente en el plano del liderazgo y la mejora de los profesores en los diferentes procesos y tareas de la educación superior este trabajo se propuso como objetivo proponer vías para la configuración de centros educativos superiores como comunidades profesionales de instrucción que puedan posibilitar el aprendizaje a través del trabajo conjunto.

\section{Fundamentación teórica}

Cualquier discurso sobre el liderazgo y mejora de la educación superior es dependiente de una comprensión de la característica de los centros educativos superiores como organizaciones y de una teoría del cambio educativo. Como es público, las prohibiciones que presentan las teorías propuestas del liderazgo provienen de haber transferido estilos, modos eficientes de liderazgo a los centros educativos, procedentes de otros contextos sean políticos, industriales entre otros, ignorando su especificidad como organizaciones educativas. Si las formas tradicionales de gestión basadas en el control burocrático y en la autoridad técnico-racional no son por sí mismas suficientes, ni legitimables; es necesario ampliar la noción de liderazgo para incluir en ella la autoridad moral y profesional, el acuerdo y colegialidad, y el liderazgo múltiple de los profesores.

El reto crítico es lograr un desarrollo institucional de los centros educativos superior como unidades de cambio en que el liderazgo está confuso y apropiado por el conjunto de miembros de la institución superior, con el riesgo de que los líderes naturales, bajo la "aureola" moral (Sergiovanni, 1992), se nos conviertan en carismáticos, dado que no podemos aceptar unas condiciones formales de igualdad. Por eso los problemas que plantea un enfoque crítico del liderazgo y mejora de la educación superior son, entre otros; El desafío de la difusividad: eliminar el liderazgo formal para difuminarlo entre todos, en la práctica puede dar lugar a que florezcan liderazgos morales incontrolados, según las cualidades carismáticas de imponerse a los restantes miembros del grupo. 
Rev. SINAPSIS, Vol. 5, N² 2, Diciembre 2014

ISSN $1390-7832$

Lograr una comunidad ideal de comunicación, en que todos los miembros tengan igual grado de competencia comunicativa para consensuar constructivamente valores e intereses de lo que deba ser el centro educativo superior, es más bien un ideal contra fáctico que una utópica ejecución real.

La capacidad para mejorar una institución de educación superior depende, de manera relevante, de equipos directivos con liderazgo que contribuyan a dinamizar, apoyar, animar y, que aprenda a desarrollarse, ayudando a construir la capacidad interna de mejora.

Según, (McKinsey, Barber y Mourshed, 2007) y la propia OCDE (Pont, Nusche y Moorman, 2008) sitúan el liderazgo educativo como el segundo factor interno a la educación que más relevancia tiene en los logros de aprendizaje, tras la acción docente de su profesorado. Se pretende un quiebre en atribuciones y competencias de los directivos, de forma que potencie su incidencia en la mejora de los aprendizajes de los respectivos establecimientos de educación superior. Acudir a lo que la investigación internacional pone de manifiesto puede contribuir decididamente para señalar modos de actuar en esta área estratégica.

Como dicen Stoll y Temperley (2009) plantean que los líderes escolares sólo es posible que influyan $r$ en los resultados de los estudiantes si hay existencia de autonomía suficiente para tomar decisiones importantes acerca del currículum, la selección y formación de maestros; además, sus principales áreas de responsabilidad deberán concentrarse en mejorar el aprendizaje de los y las estudiantes. Los países optan cada vez más por la toma de decisiones descentralizadas y por equilibrar ésta con una mayor centralización de los regímenes de rendición de cuentas, como las pruebas estandarizadas.

El liderazgo se tiene que dirigir, por eso, a transformar los modos habituales de enseñanza en nuevos escenarios de aprendizaje. El equipo directivo tiene que jugar una función hábil entre la transacción con los colegas y las necesidades de transformación que pueden demandarse desde otras instancias.

Al respecto, dice Elmore (2008): Para un director, la práctica de mejora consiste, en gran medida, en hacer visible lo que no se ve. La mayor parte de personas que ocupan un puesto dirigente en la educación superior están más o menos socializadas en una cultura relativamente disfuncional. Esta socialización consiste, entre otras cosas, en aprender a 
Rev. SINAPSIS, Vol. 5, No 2, Diciembre 2014

considerar que la mayoría de aspectos de la educación superior y de su cultura como adquisiciones establecidas, se concentran sobre un pequeño número de cosas que la cultura define como posibles. Para que los directivos aprendan a gestionar activamente el proceso de mejora, es preciso ponerse al día, analizar y modificar todas las reglas, normas y convenciones implícitas que constituyen obstáculos para la acción.

\section{Materiales y Métodos}

Para la realización de este trabajo se utilizó el apoyo documental, donde se consultaron diferentes publicaciones realizadas por autores sobre el tema que fueron la base para la fundamentación teórica de la investigación. Se realizaron además encuestas docentes y directivas que laboran en este nivel de enseñanza, material que ayudó a probar los resultados obtenidos.

La muestra de este estudio ha estado compuesta por 85 docentes y personal administrativo de las diferentes universidades e institutos de la provincia de Manabí, durante el mes de enero de 2014.

\section{Análisis estadísticos y resultados}

1.- Como mide el grado de esfuerzo que estaría dispuesto a asumir atendiendo al liderazgo directivo y a la gestión del Centro de Educación Superior en el que trabaja.

\begin{tabular}{|c|lc|c|c|}
\hline Orden & Alternativas & f & $\%$ \\
\hline a) & Alto & 14 & 16,47 \\
\hline b) & Medio & 61 & 71,76 \\
\hline c) & Bajo & 10 & 11,76 \\
\hline & & $\mathbf{8 5}$ & $\mathbf{9 9 , 9 9}$ \\
\hline
\end{tabular}




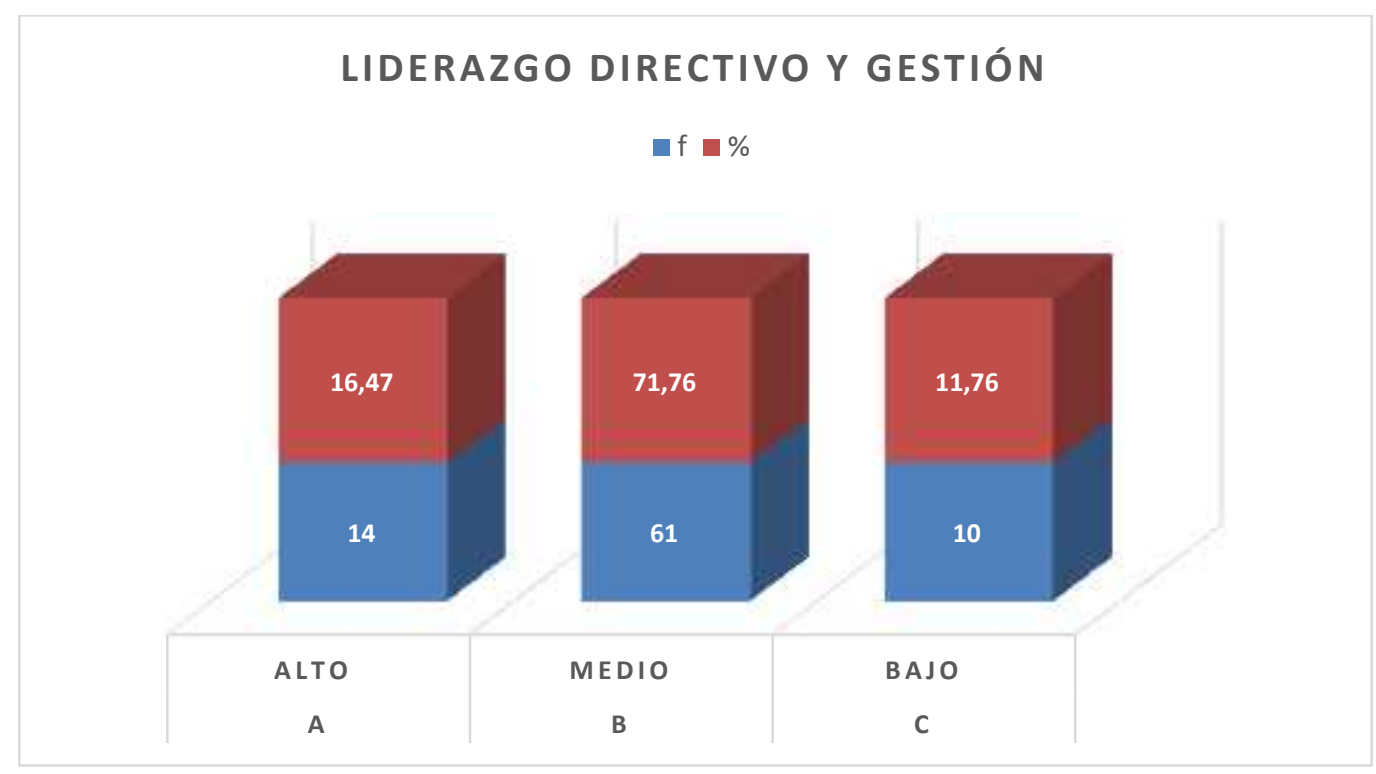

Fuente: Personal Docente y Administrativo de las Universidades e Institutos

Del total de la muestra, que corresponde a la alternativa medio, considera que el grado de esfuerzo que estaría dispuesto a asumir atendiendo al liderazgo directivo y a la gestión del Centro de Educación Superior en el que trabaja está representado por un 71,76\%; a diferencia de un $16,47 \%$ con la alternativa alto, y con la alternativa bajo se figura con un $11,76 \%$.

2.- Marque aquellos indicadores que usted considera que valoran la eficacia de los directivos para gestionar la educación superior.

\begin{tabular}{|c|l|c|c|}
\hline Orden & \multicolumn{1}{|c|}{ Alternativas } & f & \% \\
\hline a) & Los recursos económicos, materiales y las instalaciones. & 75 & 16,85 \\
\hline b) & Los documentos institucionales. & 45 & 10,11 \\
\hline c) & La implementación educativa de las TICS. & 85 & 19,10 \\
\hline d) & Los planes institucionales de acción tutorial. & 30 & 6,74 \\
\hline e) & El clima de convivencia. & 70 & 15,73 \\
\hline f) & Los apoyos para el desarrollo profesional de los docentes & 84 & 18,88 \\
\hline g) & Los procesos de evaluación, asesoramiento y supervisión & 56 & 12,58 \\
\hline & TOTAL & $\mathbf{4 4 5}$ & $\mathbf{9 9 , 9 9}$ \\
\hline
\end{tabular}




\section{INDICADORES EN LA EFICACIA DE LOS DIRECTIVOS}

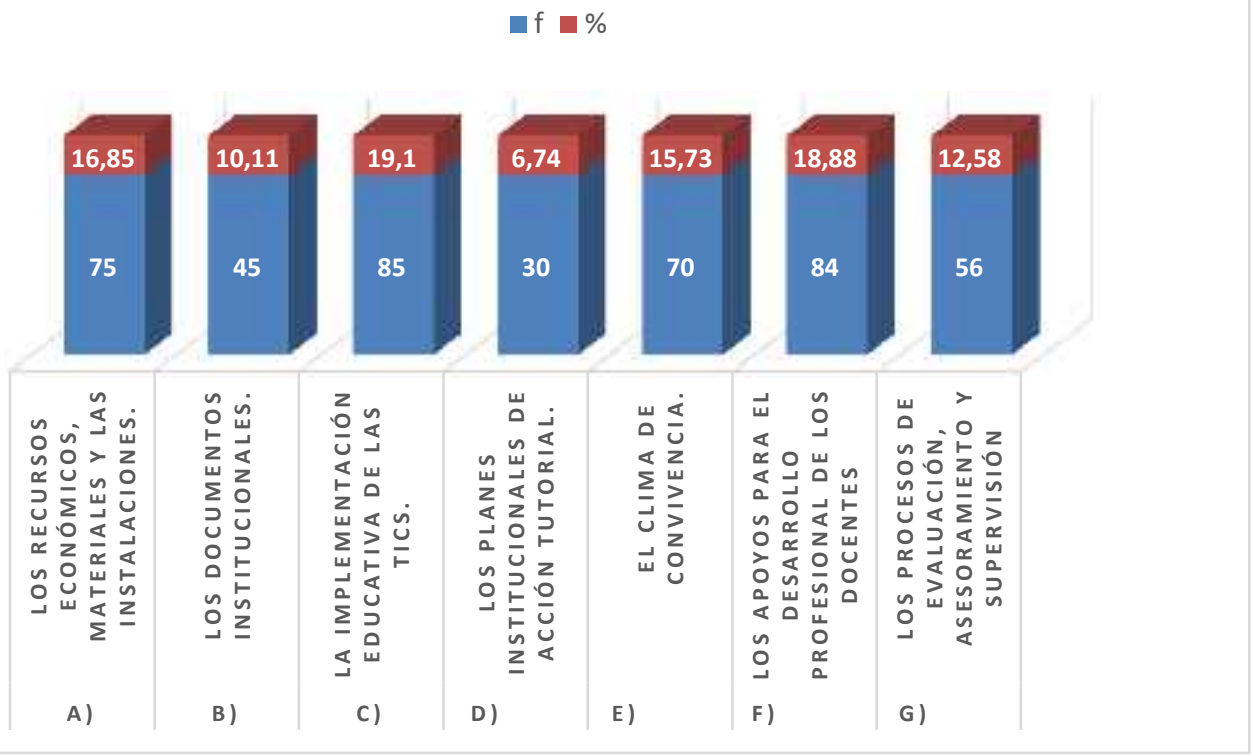

Fuente: Personal Docente y Administrativo de las Universidades e Institutos

Del total de la muestra, 85 encuestados con el 19,10\% manifestaron que la implementación educativa de las Tics es uno de los indicadores que valoran la eficacia de los directivos para gestionar la educación superior; así mismo con un 18,88\% mencionan que uno de los indicadores es el apoyo para el desarrollo profesional de los docentes; seguidamente tenemos un $16,85 \%$ que corresponde a los recursos económicos, materiales y las instalaciones que es otro indicador fundamental; continuamos con el indicador que es el clima de convivencia con un 15,73\% de aceptación; luego tenemos con un 12,58\% a los procesos de evaluación, asesoramiento y supervisión; con un $10,11 \%$ los documentos institucionales y por último con un $6,74 \%$ los planes institucionales de acción tutorial, indicador que valora la eficiencia de los directivos para gestionar la educación superior.

3.- Seleccione aquellas variables de los estilos de liderazgo que se aplican en el Centro de Educación Superior al que pertenece.

\begin{tabular}{|c|l|c|c|}
\hline Orden & \multicolumn{1}{|c|}{ Alternativas } & f & \% \\
\hline a) & Respalda la calidad de la docencia. & 42 & 13,55 \\
\hline b) & Fomenta la investigación educativa. & 50 & 16,13 \\
\hline c) & Mantiene y potencia relaciones con las organizaciones. & 46 & 14,84 \\
\hline d) & Favorece los intercambios de experiencias innovadoras. & 58 & 18,71 \\
\hline e) & Asesora y supervisa en la planificación operativa anual. & 64 & 20,64 \\
\hline f) & Gestionar los sistemas de calidad. & 50 & 16,13 \\
\hline & TOTAL & $\mathbf{3 1 0}$ & $\mathbf{1 0 0}$ \\
\hline
\end{tabular}




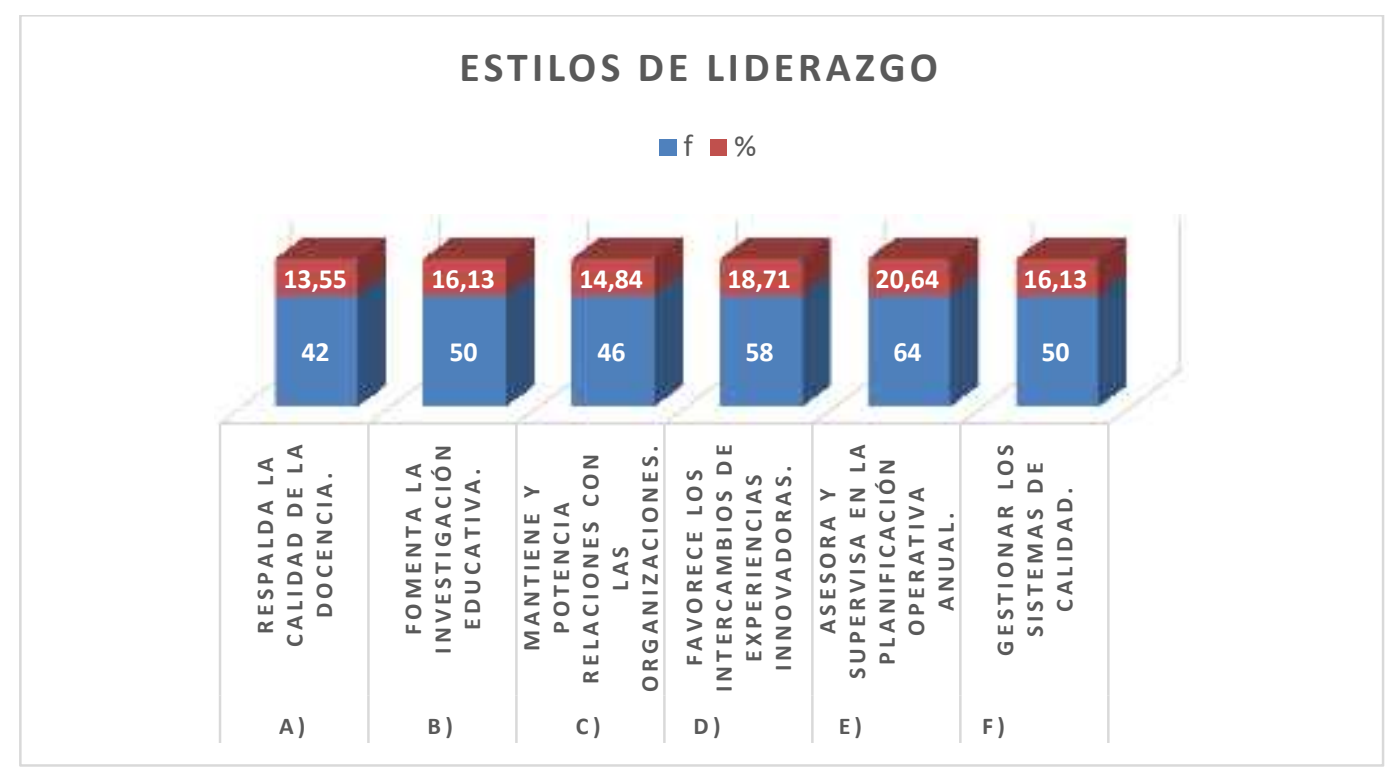

Personal Docente y Administrativo de las Universidades e Institutos

Fuente:

Del total de la muestra el 20,64\% de los encuestados mencionan que una de las variables de los estilos de liderazgo que se aplican en el Centro de Educación Superior es el asesoramiento y supervisión en la planificación operativa anual; seguidamente tenemos con un $18,71 \%$ la variable donde menciona el favorecer los intercambios de experiencias innovadoras; otra de las variables con un porcentaje de $16,13 \%$ son las alternativas b y f donde fomenta la investigación educativa y gestiona los sistemas de calidad; luego tenemos con un 14,84\% señalando los encuestados que otro estilo de liderazgo es cuando mantiene y potencia relaciones con las organizaciones; finalmente los encuestados indican que otro estilo de liderazgo es el respaldo de la calidad de la docencia con un 13,55\%.

4.- Considera usted que la falta de liderazgo provocan los fracasos en la Educación Superior.

\begin{tabular}{|c|lc|c|c|}
\hline Orden & Alternativas & f & \% \\
\hline a) & Si. & 85 & 100 \\
\hline b) & No. & 0 & 0 \\
\hline & & $\mathbf{8 5}$ & $\mathbf{1 0 0}$ \\
\hline
\end{tabular}




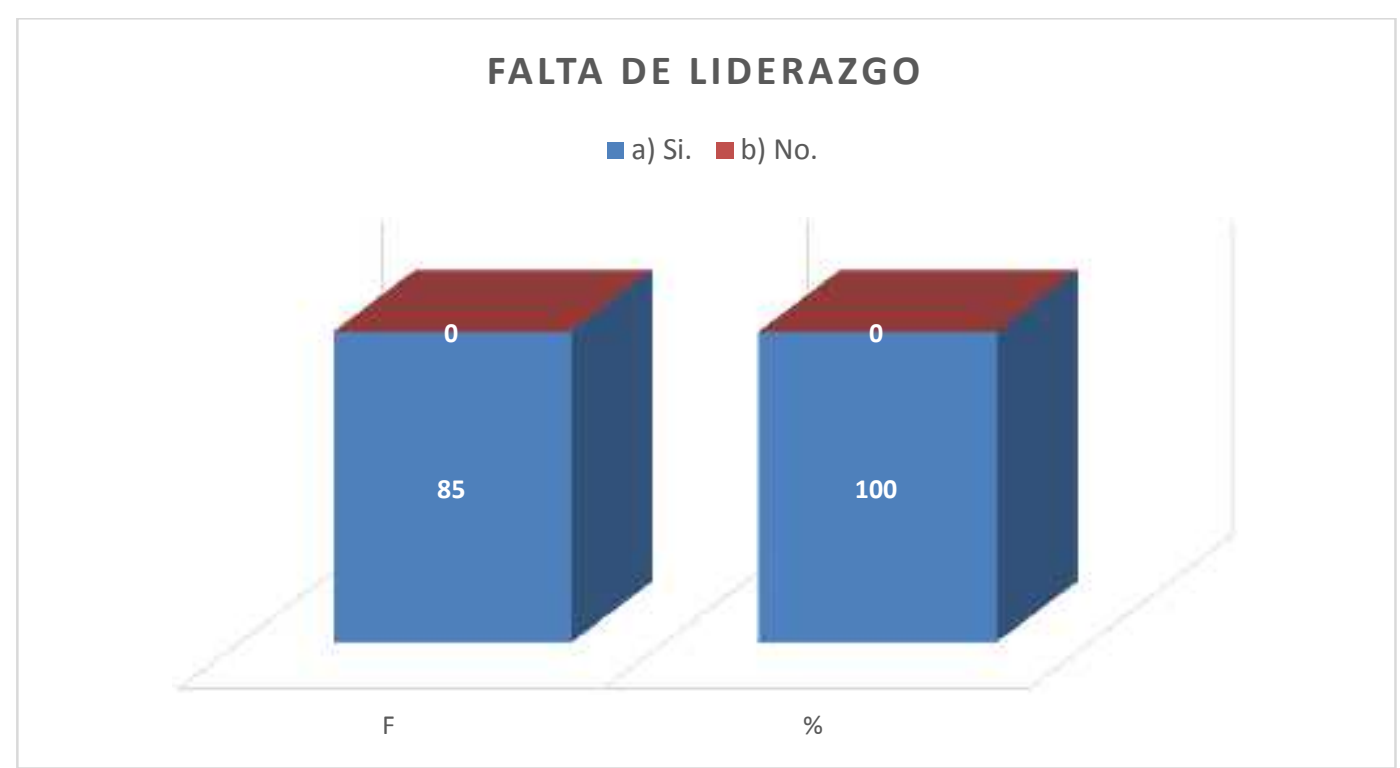

Personal Docente y Administrativo de las Universidades e Institutos

Fuente:

Del total de la muestra de los 85 encuestados todos se manifestaron por la alternativa si con el $85 \%$ que considera que la falta de liderazgo provoca los fracasos en la educación superior.

5.- ¿Cómo evalúa la existencia de liderazgo en la Educación Superior?

\begin{tabular}{|c|l|c|c|}
\hline Orden & Alternativas & f & \% \\
\hline a) & Excelente & 85 & 100 \\
\hline b) & Muy buena & 0 & 0 \\
\hline c) & Buena & 0 & 0 \\
\hline d) & Regular & 0 & 0 \\
\hline e) & Deficiente & 0 & 0 \\
\hline & & $\mathbf{8 5}$ & $\mathbf{1 0 0}$ \\
\hline
\end{tabular}

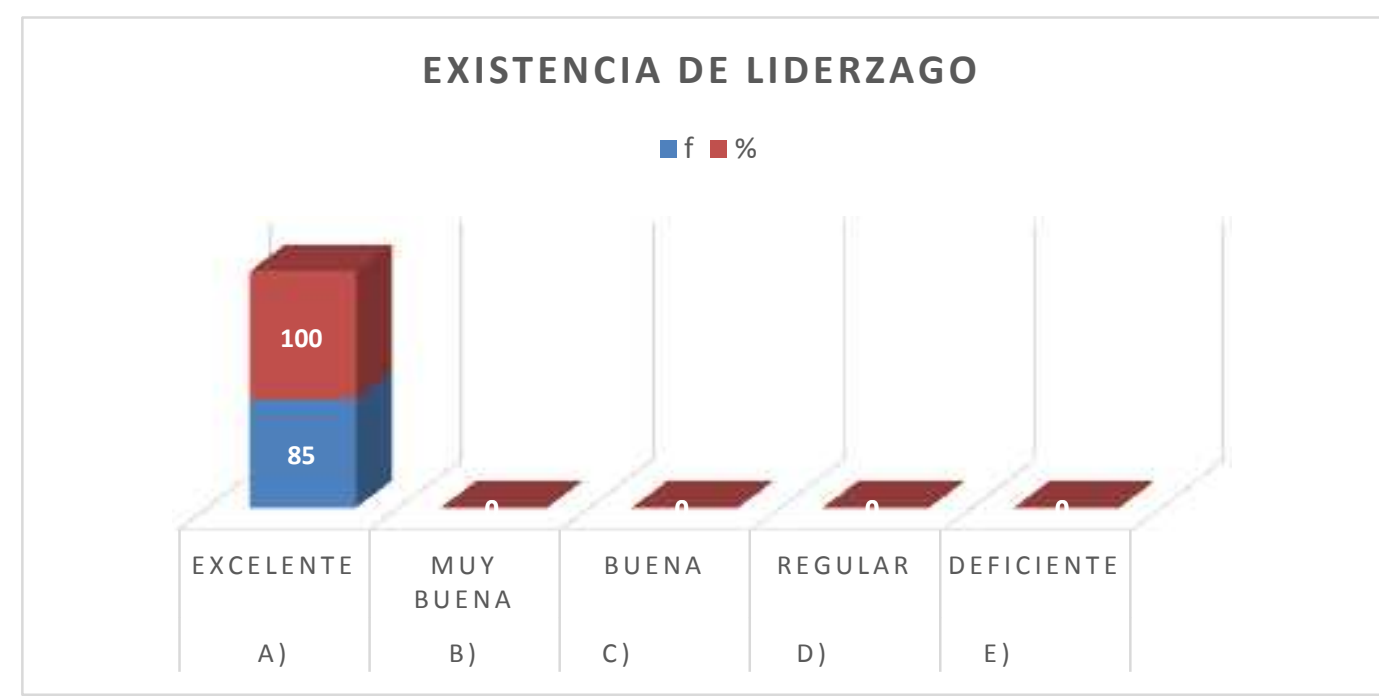

Personal Docente y Administrativo de las Universidades e Institutos

Fuente: 
Rev. SINAPSIS, Vol. 5, No 2, Diciembre 2014

ISSN $1390-7832$

Del total de la muestra el $85 \%$ de los encuestados manifiestan que la existencia de liderazgo en la educación superior es excelente.

6.- Considera usted que es prioridad la necesidad de mejorar el liderazgo en la educación superior.

\begin{tabular}{|c|lc|c|c|}
\hline Orden & \multicolumn{2}{|c|}{ Alternativas } & f & $\%$ \\
\hline a) & Totalmente & 85 & 100 \\
\hline b) & Parcialmente & 0 & 0 \\
\hline c) & De ninguna manera & 0 & 0 \\
\hline & & TOTAL & $\mathbf{8 5}$ & $\mathbf{1 0 0}$ \\
\hline
\end{tabular}

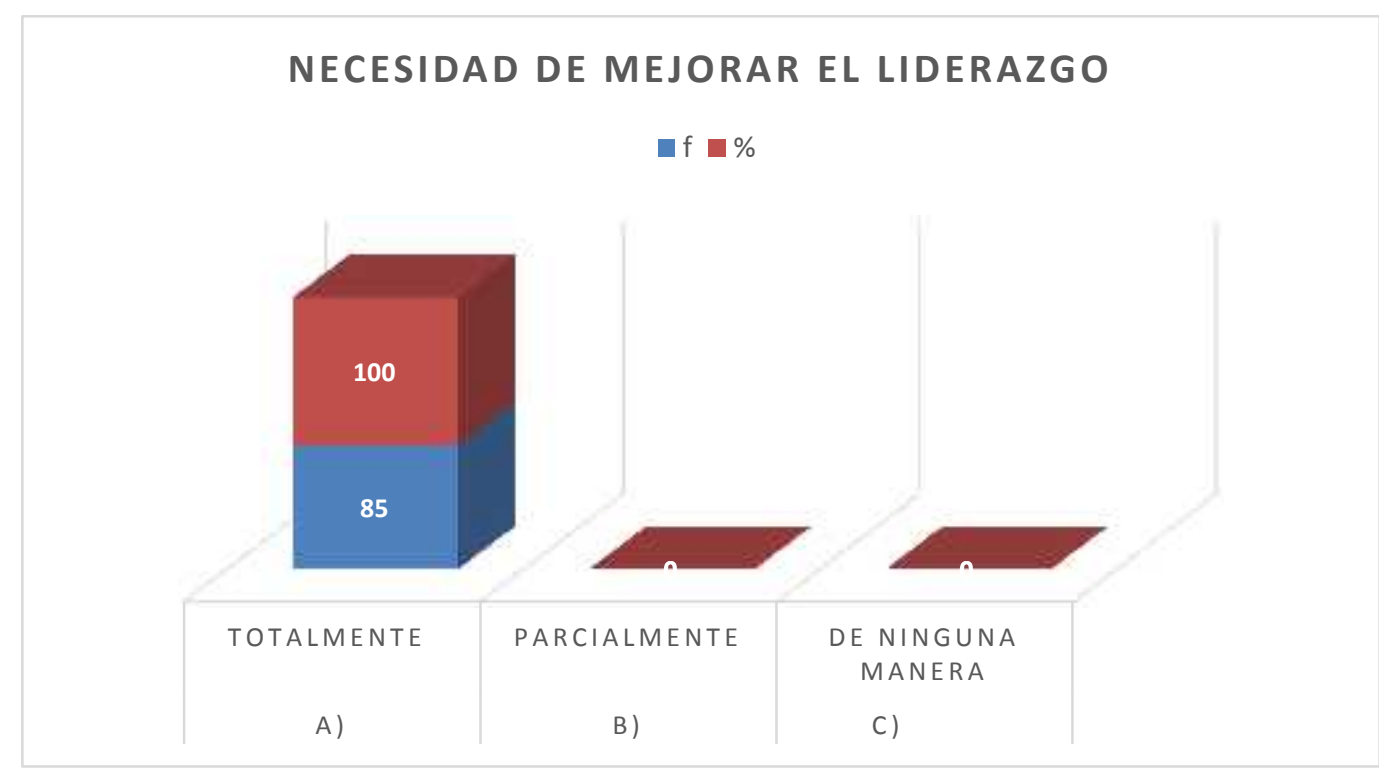

Personal Docente y Administrativo de las Universidades e Institutos

Fuente:

Del total de la muestra el $85 \%$ de los encuestados consideran que totalmente es prioridad la necesidad de mejorar el liderazgo en la Educación Superior.

\section{Discusión}

Se requiere un quiebre, en varias dimensiones, convirtiendo a este nivel en un factor catalizador del cambio de la educación superior. Dentro de la revisión crítica de las políticas de reforma de los 90 y sus resultados, la mejora docente en el aula, como ya lo apuntaba el marco para una buena dirección, demanda nuevos modos de ejercer la dirección de los establecimientos educativos superiores.

Sorprende, pues, que la política educativa haya mantenido en el olvido durante tanto tiempo a unos profesionales con un papel tan decisivo sobre los resultados de la educación superior. A 
continuación alguna conclusiones que, de acuerdo con los análisis previos, pueden sugerir propuestas de interés. En primer lugar, objetivo prioritario de las políticas educacionales en el siglo XXI es garantizar a todos los estudiantes los aprendizajes imprescindibles que les posibiliten, sin riesgo de exclusión, la integración y participación activa en la vida pública. El liderazgo y mejora de la educación superior en la enseñanza está, sin duda, para hacerlo posible. Necesitamos, pues, los mejores equipos directivos que puedan ejercer un liderazgo educativo. En segundo lugar, se precisa una formación de tercer nivel y en servicio adecuadas. Los líderes educativos necesitan capacitación específica para responder al aumento de funciones y responsabilidades, en particular sobre estrategias y resultados para el liderazgo y mejora de la educación superior.

Si la acción del liderazgo y mejora se debiera dirigir a crear contextos para el aprendizaje y las estructuras de la educación superior estos favorecen a los valores idiosincráticos aislados y aprendizaje individualista, son cambios preciosos transformacionales, a nivel organizativo, que posibilitan las acciones deseadas.

Al fin y al cabo, la capacidad de cambio de la educación superior dependerá no de una cúspide, sino de que el liderazgo y mejora de la dirección se diluya, de modo que como cualidad de la organización genere el liderazgo múltiple de los miembros y grupos, siendo por tanto algo compartido. Si queremos que los profesores asuman un papel más profesional, con funciones de liderazgo y mejora en sus respectivas áreas y ámbitos, deben asumir dirección y autoridad en sus respectivos ámbitos.

El director, en este sentido, tiene que ejercer un papel "transformador": estimular y desarrollar un clima de colegialidad, contribuir al desarrollo profesional de sus profesores, e incrementar la capacidad para resolver sus problemas. Construir una visión colectiva y situar los objetivos prácticos, creación de culturas de colaboración, altas expectativas de niveles de consecución y proveer apoyo psicológico y material al personal, son otras tantas dimensiones de estas funciones transformadoras.

\section{Conclusiones}

Se concluyó que el modelo de liderazgo pedagógico se centra en la práctica de la mejora de la calidad de la enseñanza y el rendimiento de los estudiantes, trata de liderazgo como una función distribuida más que como un rol basado en la actividad, y requiere continua 
Rev. SINAPSIS, Vol. 5, No 2, Diciembre 2014

formación y actualización de conocimientos y habilidades, tanto porque el conocimiento base de la práctica docente está cambiando constantemente como porque hay que reponer la población de los líderes actuales.

\section{Bibliografía}

1. Álvarez, F. (1998) El liderazgo de la calidad total. Escuela Española. Madrid. España.

2. Álvarez, M. (2001). El liderazgo de los procesos de mejora, en I. Cantón (Coord.). Editorial CCS. La implantación de la calidad en los centros educativos. Madrid. España.

3. Antúnez, S. (1987). El Proyecto Educativo de Centro. Graó. Barcelona. España.

4. Antúnez, S. y otros (1992). Del Proyecto Educativo a la Programación de Aula. Graó. Barcelona. España.

5. Arana, A. (2013). La compatibilidad del líder base de su influencia. Recuperado de http://www.degerencia.com/articulo/la-confiabilidad-del-liderbase-de-su-influencia el 22 de marzo de 2014.

5. Bass, B (2000). Liderazgo y organizaciones que aprenden. ICE Deusto.En Actas del III Congreso Internacional sobre Dirección de Centros Educativos, 331-361, Bilbao. España.

6. Barber, M. y Mourshed, M. (2007). Cómo hicieron los sistemas educativos con mejor desempeño para alcanzar sus objetivos.

7. Pont, B.; Nusche, D. y Moorman, H. (2008). Mejorar el liderazgo escolar. Volumen 1: política y práctica. OCDE. España.

8. Pont, B.; Nusche, D. y Hopkins, D. (eds.) 2008). Mejorar el liderazgo escolar. Volumen 2: casos prácticos sobre el liderazgo del sistema. OCDE. España. 